\title{
Tumor-infiltrating lymphocyte as a prognostic biomarker in stage IV colorectal cancer should take into account the metastatic status and operation modality
}

This article was published in the following Dove Press journal:

Cancer Management and Research

Qian-Kun Xie, ${ }^{1, *}$ Wen-Zhuo $\mathrm{He},{ }^{1, *}$ Wan-Ming $\mathrm{Hu},{ }^{2-4}$ Lin Yang,' Chang Jiang,' PengFei Kong, 'Yuan-Zhong Yang, ${ }^{2}$ Qiong Yang, 1,5 Hui-Zhong Zhang, ${ }^{2}$ Bei Zhang,' Liang-Ping Xia'

IVIP Region, State Key Laboratory of Oncology in South China, Collaborative Innovation Center for Cancer Medicine, Sun Yat-sen University Cancer Center. Guangzhou, China; ${ }^{2}$ Department of Pathology, State Key Laboratory of Oncology in South China, Collaborative Innovation Center for Cancer Medicine. SunYat-sen University Cancer Center, Guangzhou, China; ${ }^{3}$ Department of Pathology, School of Basic Medical Sciences, Southern Medical University, Guangzhou, China; ${ }^{4}$ Department of Pathology, Nanfang Hospital, Southern Medical University, Guangzhou, China; ${ }^{5}$ Department of Oncology, Sun Yat-sen Memorial Hospital, Guangzhou, China

*These authors contributed equally to this work

Correspondence: Liang-Ping Xia

State Key Laboratory of Oncology in South China, Collaborative Innovation Center for Cancer Medicine, Sun Yat-sen University Cancer Center, 65I Dongfeng East Road, Guangzhou, Guangdong 510060, China

Tel +86 2087343107

Fax +862087343392

Email xialp@sysucc.org.cn

Bei Zhang

State Key Laboratory of Oncology in South China, Collaborative Innovation Center for Cancer Medicine, Sun Yat-sen University Cancer Center, 65I Dongfeng East Road, Guangzhou, Guangdong 5I0060, P. R. China Tel +86 2087343107

Fax +86 2087343392

Email zhangbei@sysucc.org.cn
Background: Although tumor-infiltrating lymphocytes (TILs) have been understood for years as a favorable prognostic factor for colorectal cancers (CRCs) after complete surgical resection, its prognostic role in metastatic $\mathrm{CRC}(\mathrm{mCRC})$ remains poorly defined, and it is largely unknown how this prognostic benefit relates to the metastatic status and operation modality.

Materials and methods: After reviewing 2215 consecutive cases of surgically resected CRC, 332 patients newly diagnosed with stage IV CRC and treated at the Sun Yat-Sen University Cancer Center between 2009 and 2014 were included. H\&E-stained (HES) slides from surgical specimens were evaluated for the extent of TILs. The primary end point was overall survival (OS). Cox proportional hazards regression was conducted to determine the prognostic significance of TILs. All statistical tests were 2-sided.

Results: HES slides from primary tumor samples were evaluable for 302 of the 332 included cases. Among the 302 patients, 105 patients (34.8\%) were classified as high TIL, the remaining $197(65.2 \%)$ were defined as low TIL. In the univariate analysis, TILs were significantly associated with better OS $(P=0.015)$. Multivariable analysis confirmed that high TIL strongly predicted better survival (hazard ratio $=0.62,95 \%$ CI: $0.44-0.89, P=0.008$ ), independent of other patients' clinicopathological characteristics. Stratified analysis revealed a prognostic benefit of high TIL for patients in the subgroup with non-oligometastatic disease $(P=0.002), \geq 2$ metastatic organs $(P=0.006)$, and non-metastasectomy $(P=0.005)$. By contrast, oligometastatic disease, 1 metastatic organ, or metastasectomy fully abrogated the prognostic effect of TIL.

Conclusion: Our findings indicate that the level of TILs can be used to predict the outcome for patients with mCRC; however, the operation modality and the metastatic status of patients should also be taken into account.

Keywords: colorectal cancer, stage IV, tumor-infiltrating lymphocytes, metastatic status, survival

\section{Introduction}

Colorectal cancer (CRC) is a heterogeneous complex of diseases accompanied by the accumulation of distinctive genetic and epigenetic alterations. Despite more and more molecular pathways underlying CRC are identified during the last years, very few biomarkers can be used to predict survival accurately. ${ }^{1-3}$ Prognostication mainly relies on stage at diagnosis and treatment.

With the identification of the microsatellite instability (MSI) phenotype and the corresponding longer patient survival, the host immune response, notably a greater 
lymphocytic reaction, has become a research hotspot in CRC. ${ }^{4,5}$ In addition, recent clinical results obtained with immune checkpoint blockade in metastatic CRC (mCRC) provide compelling evidence that spontaneous immunosurveillance and $\mathrm{T}$ cells play an important role in the control of CRC progression. ${ }^{6}$ However, the prognostic and predictive implications of tumor-infiltrating lymphocytes (TILs) in mCRC remain poorly understood.

Some evidence has shown that patients with high TILs at any stage generally have a better prognosis than those with low TIL disease; however, these analyses included patients with predominately nonmetastatic disease. ${ }^{5,7-10}$ These studies were also restricted by the limited information on treatments and pathologic features. Finally, it remains to be determined whether the presence of TILs provides prognostic information independent of the established prognostic factors, such as treatment regimen, MSI and the number of metastasis sites involved; all these variables have been associated with prognosis and may confound each other in survival analysis. ${ }^{11,12}$

In this study, we aimed to investigate the distribution and clinical relevance of TILs in the mCRC setting. Thus, we first assessed the relationship between TIL quantity and clinical outcome as well as clinicopathologic features in a large consecutive series of mCRC patients diagnosed and treated at a single institution to analyze the prognostic effect of baseline host antitumor immunity in this setting. Second, we also explored whether the prognostic value of TIL quantity differed with respect to metastatic status and metastasectomy, considering distant metastases are a major cause of death in patients with CRC.

\section{Materials and methods Study population}

This study reviewed 2215 consecutive patients with CRC who underwent primary tumor resection at the Sun Yat-Sen University Cancer Center between 2009 and 2014. Eligibility criteria were pathologically confirmed CRC, synchronous distant metastasis at the time of diagnosis, no history of previous malignancies or concomitant primary cancer, no inflammatory bowel disease, no Lynch syndrome, availability of H\&E-stained (HES) slides from primary tumor tissues as well as complete data on clinicopathological features (age, sex, tumor size, grade, and treatment regimen), and survival. A total of 332 patients newly diagnosed with stage IV CRC met these criteria and were included in the present study. Cancers located proximal to the splenic flexure were classified as right sided; left-sided cancers included tumors arising distal to the splenic flexure and sigmoid colon, or rectal cancers.
The extent of tumor spread was determined using X-ray, CT, MRI, and/or PET preoperatively. Intraoperative findings also contributed to the determination of tumor involvement. CRC with 5 or fewer metastases was termed as oligometastatic CRC. This study was approved by the Ethics Committee of Sun Yat-Sen University Cancer Center, and written informed consent was obtained from all patients.

\section{TILs}

HES slides from primary tumor specimens for all patients were retrieved from the Institutional Pathology Archives. The extent of TILs was assessed by using a $4^{\circ}$ scale and recorded as 0 (absent), 1+ (mild), 2+ (moderate), or 3+ (marked), as described in previous reports. ${ }^{8,10}$ In this method, all mononuclear cells, including lymphocytes and plasma cells are evaluated (granulocytes and polymorphonuclear leukocytes are excluded). Sections were defined as high TIL if scored 2 or 3 and as low TIL if scored 0 or 1 . For technical reasons (e.g., low tumor area, poor staining technique, etc.), cases that could not be appropriately evaluated were designated as not evaluable. Where there was disagreement regarding TIL category between pathologists, joint reevaluation was performed to arrive at a consensus.

\section{Immunohistochemistry and assessment of mismatch repair (MMR) status}

Immunohistochemistry for the 4 most common MMR proteins was performed with the standard Envision 2-step procedure, as described in the Supplementary materials.

\section{Statistical analysis}

All statistical analyses were performed using SPSS version 20.0 (IBM Inc., Armonk, NY, USA) or Stata 12 (StataCorp LLC, College Station, TX, USA). Chi-squared test or Fisher's exact test was used to analyze the correlations between TIL infiltration and patients' clinicopathological features. Overall survival (OS) was defined as the time from the diagnosis of the cancer until any-cause death or to the last follow-up for cases that were alive. Kaplan-Meier curves and log-rank tests were performed to compare survival across groups of high TILs vs. low TILs. Unadjusted and multivariable Cox proportional hazards regression analyses were used to evaluate the association between TIL infiltration and clinical outcome as well as the corresponding hazard ratios and 95\% CIs. Multivariable models included the following baseline variables: age, sex, lymph node status, primary tumor location, T-stage, histological grade, MMR status, previous therapy, adjuvant therapy, oligometastatic status, number of metastatic organs, 
and metastasectomy. A multivariate analysis using the Cox proportional hazards regression model was performed on all variables with $P<0.05$ in a univariate analysis to determine the independent prognostic value of TILs for patient outcomes. The effect of TIL infiltration on survival outcomes was also estimated in subgroup analyses stratified by metastatic status. All the statistical tests were 2-sided and considered significant when $P$ value $\leq 0.05$.

\section{Results}

\section{Clinicopathological characteristics and association with TIL}

HES slides from primary tumor specimens were evaluable for 302 of the 332 included cases. No difference was observed in the study population (302 patients) and the non-assessable patients (Table S1).

The median patient age was 58 years (range: 18-90 years), and 196 patients $(64.9 \%)$ were male. The primary tumors were located in the rectum (65 patients, $21.5 \%$ ) or the colon (233 patients, $77.2 \%$ ). The majority of the patients had node-positive disease and 69 patients presented with a grade 3 tumor (22.8\%). One hundred and ninety-seven $(65.2 \%)$ patients had metastases confined to an organ, while $105(34.8 \%)$ patients had metastatic sites spread over at least 2 organs. Metastasectomy was performed in 58 (19.2\%) of these patients, whereas $244(80.8 \%)$ had a palliative resection of the primary cancer. Among those patients, $74.8 \%$ received chemotherapy after surgery, and 20.5\% received chemo/radiotherapy before surgery. Approximately $18.5 \%$ of the patients did not receive any systemic chemotherapy, and surgical resection was the only tumor-directed therapy.

Representative images of TIL evaluation results are shown in Figure S1. One hundred and five patients were classified as high TIL; the remaining 197 were classified as low TIL. As presented in Table 1, high TIL was significantly associated with the MMR deficiency $(P=0.02)$ and tended to be more prominent in the absence of lymphatic invasion $(P=0.06)$ or oligometastatic tumors $(P=0.05)$. No correlation with other features, especially with the type of treatment, was observed.

\section{Association of TILs with prognosis}

After a median follow-up of 27.7 months, a total of 172 (56.9\%) deaths occurred. Those patients with a high TIL experienced a significantly better OS when compared with the low TIL group (HR, 0.67; 95\% CI: 0.48-0.92; log-rank $P=0.01)$. Kaplan-Meyer survival curves are represented in Figure 1A. Age at diagnosis, primary site, operation
Table I Tumor characteristics stratified by $\mathrm{T}$ lymphocyte infiltration status

\begin{tabular}{|c|c|c|c|}
\hline \multicolumn{4}{|l|}{ N (\%) } \\
\hline \multirow[t]{2}{*}{ Characteristics } & Low TIL & High TIL & $P$-value \\
\hline & $(N=197)$ & $(N=105)$ & \\
\hline \multicolumn{4}{|l|}{ Age (years) } \\
\hline$<65$ & $148(75.13)$ & $76(72.38)$ & 0.60 \\
\hline$\geq 65$ & $49(24.87)$ & $29(27.62)$ & \\
\hline \multicolumn{4}{|l|}{ Gender } \\
\hline Male & $125(63.45)$ & 7I (67.62) & 0.47 \\
\hline Female & $72(36.55)$ & $34(32.38)$ & \\
\hline \multicolumn{4}{|l|}{ Histologic grade } \\
\hline Moderate/well differentiated & I 28 (64.97) & 77 (73.33) & 0.46 \\
\hline Poorly differentiated & $46(23.35)$ & $23(21.90)$ & \\
\hline \multicolumn{4}{|l|}{ Primary site } \\
\hline Right & $65(32.99)$ & $25(23.81)$ & 0.14 \\
\hline Left & $132(67.01)$ & $76(72.38)$ & \\
\hline \multicolumn{4}{|l|}{ T-stage } \\
\hline TI-3 & $126(63.96)$ & $59(56.19)$ & 0.25 \\
\hline $\mathrm{T} 4$ & $69(35.03)$ & $43(40.95)$ & \\
\hline \multicolumn{4}{|l|}{ Lymph node status } \\
\hline pNo & $53(26.90)$ & $39(37.14)$ & 0.06 \\
\hline $\mathrm{pN}+$ & $140(7 \mid .07)$ & $63(60.00)$ & \\
\hline \multicolumn{4}{|l|}{ MMR status } \\
\hline dMMR & $5(2.54)$ & $9(8.57)$ & 0.02 \\
\hline PMMR & $192(97.46)$ & $96(91.43)$ & \\
\hline \multicolumn{4}{|l|}{ Previous therapy } \\
\hline De novo metastatic disease & $161(81.73)$ & 79 (75.24) & 0.18 \\
\hline Previous (neo)adjuvant & $36(18.27)$ & $26(24.76)$ & \\
\hline \multicolumn{4}{|l|}{ Oligometastatic status } \\
\hline Yes & $80(40.61)$ & $55(52.38)$ & 0.05 \\
\hline No & $117(59.39)$ & $50(47.62)$ & \\
\hline \multicolumn{4}{|l|}{ No. of metastatic organs } \\
\hline 1 & $124(62.94)$ & $73(69.52)$ & 0.25 \\
\hline$\geq 2$ & $73(37.06)$ & $32(30.48)$ & \\
\hline
\end{tabular}

Note: $P$-values were based on $\chi^{2}$ or Fisher's exact test, as appropriate.

Abbreviations: dMMR, deficient mismatch repair; pMMR, proficient mismatch repair; TIL, tumor-infiltrating lymphocyte.

modality, lymph node status, oligometastatic status, number of metastatic organs, and adjuvant therapy were the other parameters associated with outcome.

Multivariable analysis confirmed that high TILs (HR, 0.62; 95\% CI: $0.44-0.89, P=0.008$ ) were associated with a statistically significant reduction in all-cause mortality (Table 2).

\section{Stratified analysis of the correlation between TILs and OS based on metastatic lesion}

As distant metastases are a major cause of death in patients with CRC, we further evaluated whether TIL had a different prognostic impact in the group of patients stratified by metastatic lesion, including oligometastatic status, number of metastatic organs, and metastasectomy. Kaplan-Meyer 


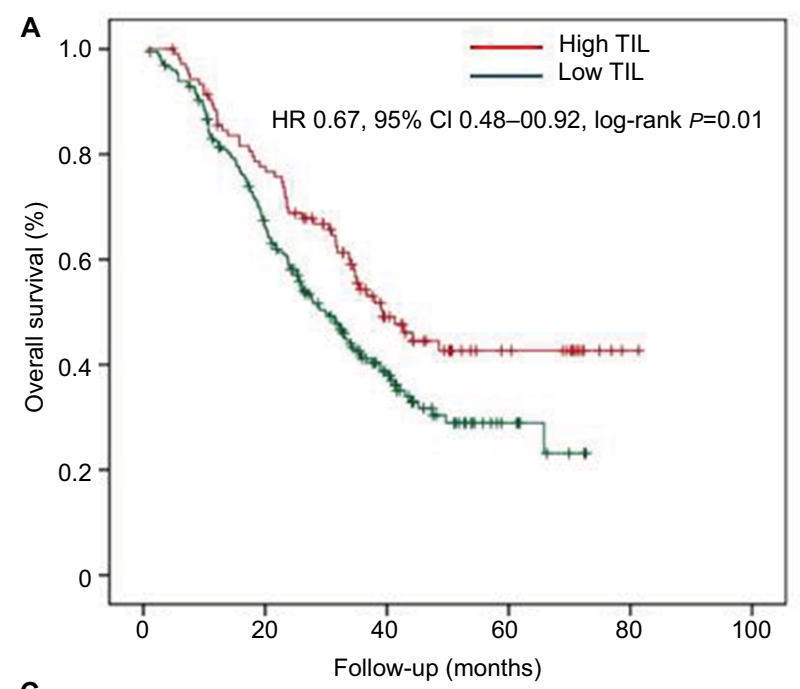

C

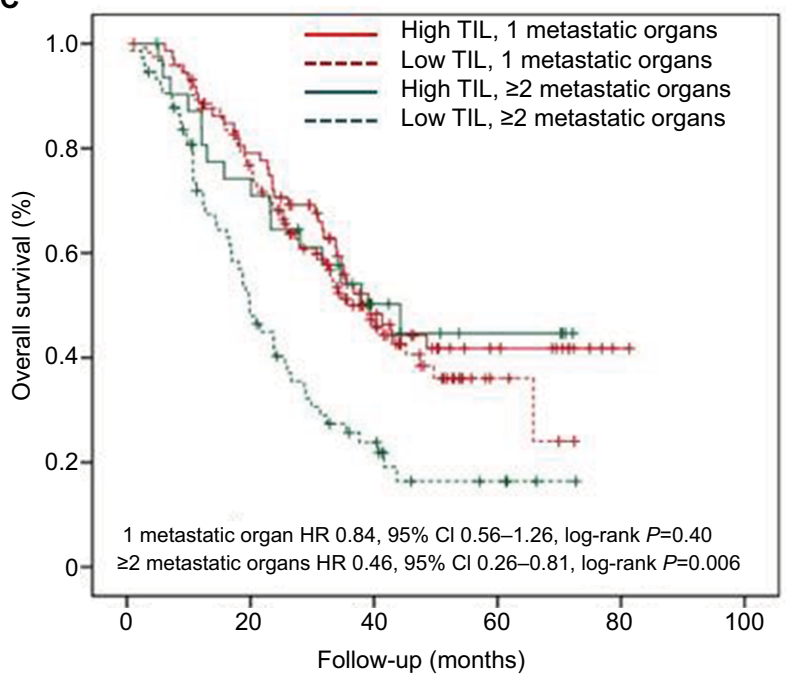

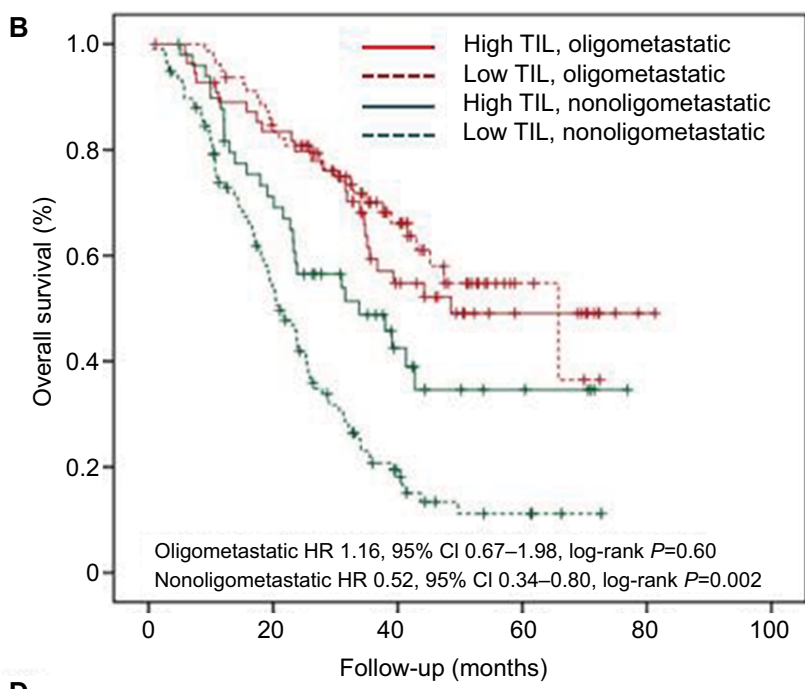

D

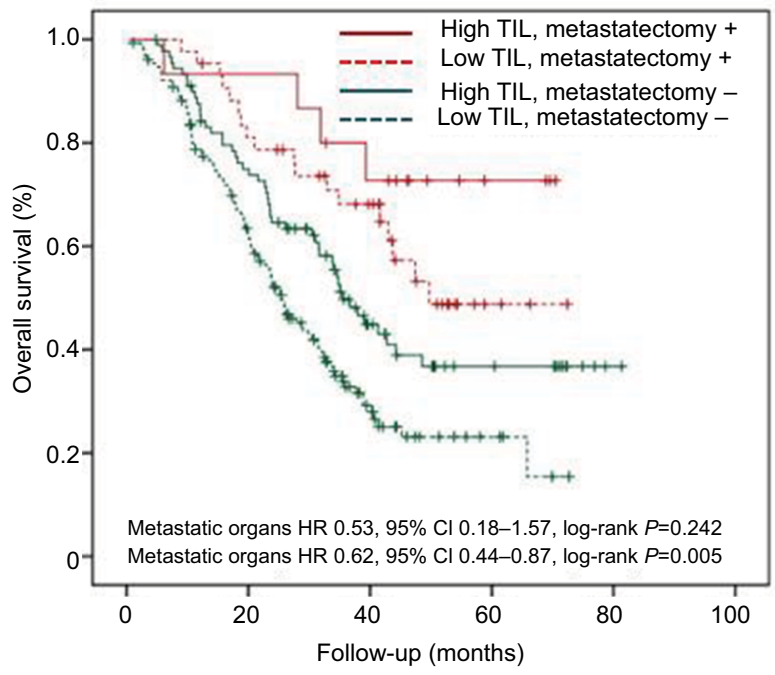

Figure I Prognostic value of TIL.

Notes: Estimated Kaplan-Meier curves of overall survival for all patients (A) and in patients grouped by TIL level and oligometastatic status (B), in patients grouped by TIL level and number of metastatic organs (C) and in patients grouped by TIL level and metastasectomy (D). All statistical tests were two-sided.

Abbreviations: HR, hazard ratio; TIL, tumor-infiltrating lymphocyte.

survival curves in each subgroup according to TIL are represented in Figure 1B-D. TIL was associated with a major prognostic value in patients with non-oligometastatic disease $(P=0.002), \geq 2$ metastatic organs $(P=0.006)$, and non-metastasectomy $(P=0.005)$. In contrast, in the cohort with oligometastatic disease $(P=0.60), 1$ metastatic organ ( $P=0.40)$, or metastasectomy $(P=0.24)$, we did not detect any significant associations between OS and level of TILs. For visual purposes, forest plots for the independent prognostic effect of TIL adjusted by other parameters in each subgroup are reported in Figure 2.

The same results were obtained with a subgroup analysis, excluding patients who received chemo/radiotherapy before surgery (Table S2).

\section{Discussion}

To the best of our knowledge, this study is the first to present the prevalence and clinical relevance of TILs in the initial stage IV CRC setting, investigating a large series and consecutive patients diagnosed and treated at a single institution. We identified a significant association between increased TILs score and improved OS in this population, independent of other patient characteristics. Our report is also unique in that we analyzed the influence of metastatic status and metastasectomy on the prognostic effects of immune infiltrates. We found that the level of TILs is only of prognostic benefit when metastases are extensive or curative-intent surgery cannot be achieved. Our data strongly indicate the clinical relevance of antitumor immunity in stage IV CRC. 
Table 2 Association of TIL with prognosis (overall survival) in the whole study population

\begin{tabular}{|c|c|c|c|c|}
\hline \multirow[t]{2}{*}{ Variable } & \multicolumn{2}{|c|}{ Univariate analysis } & \multicolumn{2}{|c|}{ Multivariate analysis } \\
\hline & HR (95\% Cl) & $P$-value & HR (95\% Cl) & $P$-value \\
\hline \multicolumn{5}{|l|}{ Age (years) } \\
\hline$<65$ & - & 0.004 & - & 0.008 \\
\hline$\geq 65$ & $1.620(1.16-2.26)$ & & $1.60(1.13-2.27)$ & \\
\hline \multicolumn{5}{|l|}{ Gender } \\
\hline Male & - & 0.55 & & \\
\hline Female & $\mathrm{I} .098(0.8 \mathrm{I}-\mathrm{I} .50)$ & & & \\
\hline \multicolumn{5}{|l|}{ Histologic grade } \\
\hline Moderate/well differentiated & - & 0.42 & & \\
\hline Poorly differentiated & $1.16(0.81-1.67)$ & & & \\
\hline \multicolumn{5}{|l|}{ Primary site } \\
\hline Right & - & 0.045 & - & 0.37 \\
\hline Left & $0.72(0.53-0.99)$ & & $0.85(0.60-1.21)$ & \\
\hline \multicolumn{5}{|l|}{ T-stage } \\
\hline TI-3 & - & 0.11 & & \\
\hline $\mathrm{T} 4$ & $1.29(0.95-1.75)$ & & & \\
\hline \multicolumn{5}{|l|}{ Lymph node status } \\
\hline $\mathrm{pN} 0$ & - & 0.004 & - & 0.04 \\
\hline $\mathrm{pN}+$ & $1.67(1.18-2.37)$ & & $1.48(1.02-2.13)$ & \\
\hline \multicolumn{5}{|l|}{ MMR status } \\
\hline dMMR & - & 0.10 & & \\
\hline PMMR & I.89 (0.88-4.05) & & & \\
\hline \multicolumn{5}{|l|}{ Previous therapy } \\
\hline De novo metastatic disease & - & 0.15 & & \\
\hline Previous (neo)adjuvant & $0.75(0.5 \mathrm{I}-\mathrm{I} . \mathrm{II})$ & & & \\
\hline \multicolumn{5}{|l|}{ Adjuvant chemotherapy } \\
\hline Negative & - & 0.01 & - & 0.003 \\
\hline Positive & $0.64(0.46-0.90)$ & & $0.58(0.4 \mathrm{I}-0.83)$ & \\
\hline \multicolumn{5}{|l|}{ Metastasectomy } \\
\hline- & - & $<0.001$ & - & 0.02 \\
\hline+ & $0.40(0.26-0.63)$ & & $0.53(0.32-0.89)$ & \\
\hline \multicolumn{5}{|l|}{ Oligometastatic status } \\
\hline No & - & $<0.001$ & - & $<0.001$ \\
\hline Yes & $0.35(0.25-0.48)$ & & $0.479(0.33-0.68)$ & \\
\hline \multicolumn{5}{|l|}{ No. of metastatic organs } \\
\hline I & - & 0.001 & - & 0.08 \\
\hline$\geq 2$ & $1.68(1.24-2.28)$ & & $1.34(0.96-1.87)$ & \\
\hline \multicolumn{5}{|l|}{ TILs } \\
\hline Low & - & 0.02 & - & 0.008 \\
\hline High & $0.67(0.48-0.92)$ & & $0.624(0.44-0.89)$ & \\
\hline
\end{tabular}

Note: All statistical tests were two-sided.

Abbreviations: dMMR, deficient mismatch repair; HR, hazard ratio; MMR, mismatch repair; pMMR, proficient mismatch repair; TIL, tumor-infiltrating lymphocyte.

Recent cumulative immunosurveillance data suggested that a dynamic and complex interaction exists between immune cells and tumor cells, and this interaction tightly controls tumor progression. ${ }^{13}$ The presence of abundant TILs is associated with a favorable prognosis for various human solid tumors, including CRC., ${ }^{5,-10,14-16}$ However, there are very few reported data on the survival impact and clinical relevance of TILs in patients with mCRC. Here, we showed that the presence of TILs is strongly associated with improved prognosis. Of note, node-negative tumors tend to have more TILs than node-positive tumors, which is in line with the observation in breast cancer and melanoma. ${ }^{17,18}$ Interestingly, for the first time, we also found that the amount of TILs in oligometastatic CRC tends to be much higher ( $40.7 \%$ in our series) than that found in the more invasive subtype (roughly 29.9\%). These data suggest that TILs in $\mathrm{mCRC}$ represents a potent stimulator of the immune system, and the alterations in overall immune function may underlie risk for tumor spread in $\mathrm{mCRC}$ patients.

Complete resection of metastatic lesions in selected patients substantially improves survival rates and long-term 


\section{Subgroup}

Oligometastatic status

Yes

No

No. of metastatic organs

1

$\geq 2$

Metastasectomy

Yes

No

Overall n

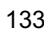

169

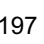

197

105

58

244

302
HR (95\% Cl)

$1.14(0.64-2.02)$

$0.56(0.36-0.87)$

$0.78(0.52-1.18)$

$0.42(0.24-0.76)$

$0.64(0.22-1.91)$

$0.62(0.43-0.89)$

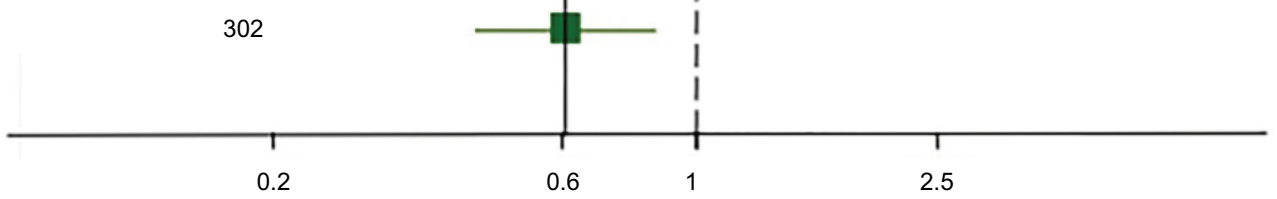

Figure 2 Prognostic value of TILs and effect of metastatic status.

Notes: Forest plots show the HR for high lymphocytic infiltration according to oligometastatic status, number of metastatic organs, and metastasectomy. These HRs were derived with a Cox regression model. Overall HRs were adjusted for other clinicopathological factors. The solid vertical line indicates an HR of 0.608 , which is the value for all patients, and the dashed vertical line indicates an HR of 1.00 , which is the null-hypothesis value.

Abbreviations: HR, hazard ratio; TIL, tumor-infiltrating lymphocyte.

survival can be expected. ${ }^{19}$ Therefore, not surprisingly, patients in the radical resection or oligometastases cohort showed a better prognosis in general. However, the heterogeneous prognostic effect of TILs according to oligometastatic status, metastatic site, and metastasectomy deserve further discussion. In contrast to the traditional immune tumor control hypothesis, one would expect that the effect of TIL infiltration on prognosis benefit could be maximized when tumor burden is small; our data indicate that TILs are of prognostic benefit only when metastases are extensive or when radical excision cannot be achieved. Nevertheless, the phenomenon is not completely surprising, especially if one considers that TILs are a robust predictive adjunct in invasive breast cancer, but their clinical role in ductal carcinoma in situ has not been observed. ${ }^{20,21}$ The absence of prognostic effect of TIL for limited metastasis represented by oligometastatic status suggests that immune aspects do not represent the key feature of this cancer type. Yet, little is known so far about the molecular basis for programmed polymetastatic or oligometastatic spread. It is reasonable to speculate that genetic properties of lesions with an oligometastatic phenotype were different from lesions "programmed" for polymetastatic dissemination. ${ }^{22}$ The potential difference in tumor biology may contribute to diversity in the immune microenvironment, including the severity of functional impairment of tumor-specific $\mathrm{T}$ cells and sensitivity of tumor cells to attack by cytotoxic $\mathrm{T}$ cells. Thereby, the heterogeneous prognostic effect of TILs can be expected. Besides, the multifaceted interplay between anti- or pro-tumoral leukocytes and mediators in the tumor microenvironment should not be ignored. Many studies indicate that the recruitment of immunosuppressive cells, such as immunoregulatory myeloid cells, regulatory $\mathrm{T}$ cells, Thelper 17 cells, and regulatory B cells to the primary tumor site protects cancer cells from being killed by cytotoxic cells and this increases the probability of tumor cell survival and dissemination. Accordingly, a high content of immunosuppressive cells may point toward poor survival. ${ }^{23}$ However, the relative densities of specific subpopulations of these lymphoid cells are almost indistinguishable in $\mathrm{H} \& \mathrm{E}$ routine slides. Therefore, TILs evaluation seems premature, disregarding TILs evaluation in the setting of limited metastasis.

The finding that TILs can identify a subset of patients unamenable to curative-intent surgery with excellent outcome has several implications in patient stratification and precise treatment. Although a number of retrospective studies have suggested that primary tumor resection is safe and associated with better outcome in mCRC patients, ${ }^{24-27}$ to date, there have been no effective factors to identify surrogates for palliative 
resection in the $\mathrm{mCRC}$ setting. Patient selection for palliative resection is only based on tumor dissemination, comorbidity, and performance status. In the present study, patients with a high TIL score presented a favorable outcome for extensive metastatic lesions. In other words, in the presence of adverse prognostic factors, a high lymphocytic infiltration could distinguish a group of patients to be good candidates to receive palliative resection. On the other hand, even in the presence of favorable prognostic factors, a low lymphocytic infiltration could distinguish a group of patients with very poor survival, who would not benefit from the palliative resection of primary tumor. Thus, TIL score could individualize more precise treatment strategies of $\mathrm{mCRC}$ and optimize cost-effectiveness ratio.

Although our study is considerably strengthened by the large sample size, detailed follow-up time and data on cancer treatment, it is not without limitations. In this study, cases were excluded if there was non-assessable archival material for full pathological analysis. However, the clinical features of the excluded patients did not differ significantly from those included in the study. In contrast to breast cancer, there is no current consensus for evaluating the level of TILs in CRC. ${ }^{28}$ Although our study provides a simple and readily available method of interpreting TILs using full-face HES sections, the methodology for assessment of TILs in CRC needs to be standardized in future, to make it practical for routine use.

\section{Conclusion}

We provide, for the first time, a comprehensive evaluation of TILs in a large and well-characterized cohort of mCRC patients. Our analyses demonstrate that TILs have a strong prognostic effect in patients with $\mathrm{mCRC}$, and metastatic status and metastasectomy should also be taken into account. This easy-to-collect information could be of great value in clinical decision making. Further studies characterizing the landscape and prognostic effect of TIL subpopulations are warranted and may provide more precise biologic significance of immune response in $\mathrm{mCRC}$.

\section{Acknowledgments}

We would like to acknowledge the assistance of the colleagues at State Key Laboratory of Oncology in South China and the Department of Pathology, Sun Yat-sen University Cancer Center. This work was supported by grants from the National Natural Science Foundation of China (81272641). The authenticity of this article has been validated by uploading the key raw data onto the Research Data Deposit public platform (www.researchdata.org.cn), with the approval RDD number as RDDA2018000527.

\section{Disclosure}

The authors report no conflicts of interest in this work.

\section{References}

1. Markowitz SD, Bertagnolli MM. Molecular origins of cancer: molecular basis of colorectal cancer. $N$ Engl J Med. 2009;361(25):2449-2460.

2. Van Schaeybroeck S, Allen WL, Turkington RC, Johnston PG. Implementing prognostic and predictive biomarkers in CRC clinical trials. Nat Rev Clin Oncol. 2011;8(4):222-232.

3. George B, Kopetz S. Predictive and prognostic markers in colorectal cancer. Curr Oncol Rep. 2011;13(3):206-215.

4. Galon J, Mlecnik B, Marliot F, et al. Validation of the Immunoscore (IM) as a prognostic marker in stage I/II/III colon cancer: results of a worldwide consortium-based analysis of 1,336 patients. J Clin Oncol. 2016;34(15 suppl):3500.

5. Rozek LS, Schmit SL, Greenson JK, et al. Tumor-infiltrating lymphocytes, Crohn's-like lymphoid reaction, and survival from colorectal cancer. J Natl Cancer Inst. 2016;108(8).

6. Khalil DN, Smith EL, Brentjens RJ, Wolchok JD. The future of cancer treatment: immunomodulation, CARs and combination immunotherapy. Nat Rev Clin Oncol. 2016;13(5)273-290.

7. Svennevig JL, Lunde OC, Holter J, Bjørgsvik D. Lymphoid infiltration and prognosis in colorectal carcinoma. Br J Cancer. 1984;49(3): 375-377.

8. Ropponen KM, Eskelinen MJ, Lipponen PK, Alhava E, Kosma VM Prognostic value of tumour-infiltrating lymphocytes (TILs) in colorectal cancer. J Pathol. 1997;182(3):318-324.

9. Galon J, Costes A, Sanchez-Cabo F, et al. Type, density, and location of immune cells within human colorectal tumors predict clinical outcome. Science. 2006;313(5795):1960-1964.

10. Klintrup K, Makinen JM, Kauppila S, et al. Inflammation and prognosis in colorectal cancer. Eur J Cancer. 2005;41:2645-2654.

11. Qiu M, Hu J, Yang D, Cosgrove DP, Xu R. Pattern of distant metastases in colorectal cancer: a SEER based study. Oncotarget. 2015;6:38658-38666.

12. Hugen N, van de Velde CJ, de Wilt JH, Nagtegaal ID. Metastatic pattern in colorectal cancer is strongly influenced by histological subtype. Ann Oncol. 2014;25:651-657.

13. de Visser KE, Eichten A, Coussens LM. Paradoxical roles of the immune system during cancer development. Nat Rev Cancer. 2006;6:24-37.

14. Baldan V, Griffiths R, Hawkins RE, et al. Efficient and reproducible generation of tumour-infiltrating lymphocytes for renal cell carcinoma. Br J Cancer. 2015;112:1510-1518.

15. Kang BW, Seo AN, Yoon S, et al. Prognostic value of tumor-infiltrating lymphocytes in Epstein-Barr virus-associated gastric cancer. Ann Oncol. 2016;27:494-501.

16. Denkert C, Loibl S, Noske A, et al. Tumor-associated lymphocytes as an independent predictor of response to neoadjuvant chemotherapy in breast cancer. J Clin Oncol. 2010;28:105-113.

17. Zuckerman NS, Yu H, Simons DL, et al. Altered local and systemic immune profiles underlie lymph node metastasis in breast cancer patients. Int J Cancer. 2013;132:2537-2547.

18. Taylor RC, Patel A, Panageas KS, Busam KJ, Brady MS. Tumorinfiltrating lymphocytes predict sentinel lymph node positivity in patients with cutaneous melanoma. J Clin Oncol. 2007;25:869-875.

19. De Haas RJ, Wicherts DA, Andreani P, et al. Impact of expanding criteria for resectability of colorectal metastases on short- and long-term outcomes after hepatic resection. Ann Surg. 2011;253:1069-1079.

20. Loi S, Michiels S, Salgado R, et al. Tumor infiltrating lymphocytes are prognostic in triple negative breast cancer and predictive for trastuzumab benefit in early breast cancer: results from the FinHER trial. Ann Oncol. 2014;25:1544-1550.

21. Pruneri G, Lazzeroni M, Bagnardi V, et al. The prevalence and clinical relevance of tumor-infiltrating lymphocytes (TILs) in ductal carcinoma in situ of the breast. Ann Oncol. 2017;28:321-328. 
22. Uppal A, Ferguson MK, Posner MC, Hellman S, Khodarev NN, Weichselbaum RR. Towards a molecular basis of oligometastatic disease: potential role of micro-RNAs. Clin Exp Metastasis. 2014;31:735-748.

23. Kitamura T, Qian BZ, Pollard JW. Immune cell promotion of metastasis. Nat Rev Immunol. 2015;15:73-86.

24. Kim YW, Kim IY. The role of surgery for asymptomatic primary tumors in unresectable stage IV colorectal cancer. Ann Coloproctol. 2013;29:44-54.

25. Scheer MG, Sloots CE, van der Wilt GJ, Ruers TJ. Management of patients with asymptomatic colorectal cancer and synchronous irresectable metastases. Ann Oncol. 2008;19:1829-1835.
26. He WZ, Rong YM, Jiang C, et al. Palliative primary tumor resection provides survival benefits for the patients with metastatic colorectal cancer and low circulating levels of dehydrogenase and carcinoembryonic antigen. Chin J Cancer. 2016;35:58.

27. Tarantino I, Warschkow R, Worni M, et al. Prognostic relevance of palliative primary tumor removal in 37,793 metastatic colorectal cancer patients: a population-based, propensity score-adjusted trend analysis. Ann Surg. 2015;262:112-120.

28. Salgado R, Denkert C, Demaria S, et al. The evaluation of tumorinfiltrating lymphocytes (TILs) in breast cancer: recommendations by an International TILs Working Group 2014. Ann Oncol. 2015;26:259-271. 


\section{Supplementary materials}

\section{Immunohistochemistry and assessment of mismatch repair (MMR) status}

Briefly, after the specimens were dewaxed, hydrated, and washed, endogenous peroxidase was blocked $\left(0.3 \% \mathrm{H}_{2} \mathrm{O}_{2}\right.$ for $10 \mathrm{~min}$ ), and microwave antigen retrieval was performed in Tris-EDTA (pH 9.0). The slides were then incubated with blocking serum at room temperature for $30 \mathrm{~min}$ and with the primary antibody at $4{ }^{\circ} \mathrm{C}$ overnight. The sections were serially rinsed, incubated with secondary antibodies, and visualized with diaminobenzidine following counterstaining with hematoxylin. The primary antibodies against the following proteins were used: MLH1 (1:50; Beijing Zhong Shan-Golden Bridge Biological Technology, Beijing, China), PMS2 (1:50; Beijing Zhong Shan-Golden Bridge Biological Technology, Beijing, China), MSH2 (1:50; Beijing Zhong Shan-Golden Bridge
Biological Technology, Beijing, China), and MSH6 (1:50; Beijing Zhong Shan-Golden Bridge Biological Technology, Beijing, China). The PBS replaced the primary antibody as a negative control. And the known MMR-deficient colorectal carcinomas served as external negative controls. Stromal cells, non-neoplastic colonic mucosa, infiltrating lymphocytes, or the centers of lymphoid follicles were used as internal positive controls. Normal expression was defined as nuclear staining within tumor cells, while negative protein expression was defined as complete absence of nuclear staining within tumor cells with concurrent internal positive controls. Tumor with loss of MLH1/PMS2/MSH2/MSH6 protein as visualized by light microscopy was defined as MLH1/PMS2/MSH2/MSH6 negative. The procedure was routinely repeated if internal non-neoplastic tissues showed invalid negative staining. When discordance were found between the opinions of the two pathologists, agreement was reached by careful discussion.

Table SI Baseline characteristics

\begin{tabular}{|c|c|c|c|}
\hline Variable & $\begin{array}{l}\text { Patients with evaluable } \\
\text { TILs }(N=302)\end{array}$ & $\begin{array}{l}\text { Patients with non- } \\
\text { evaluable TILs }(N=30)\end{array}$ & $P$-value \\
\hline \multicolumn{4}{|l|}{ Age (years) } \\
\hline$<65$ & $232(76.8)$ & $24(75.0)$ & 0.69 \\
\hline$\geq 65$ & $70(30.2)$ & $6(25.0)$ & \\
\hline \multicolumn{4}{|l|}{ Sex, No. (\%) } \\
\hline Male & $196(64.9)$ & $18(60.0)$ & 0.59 \\
\hline Female & $106(35.1)$ & $12(40.0)$ & \\
\hline \multicolumn{4}{|l|}{ Histological grade (\%) } \\
\hline Moderate/well differentiated & $205(67.9)$ & $21(70.0)$ & 0.94 \\
\hline Poorly differentiated & $69(22.8)$ & $6(20.0)$ & \\
\hline Other or missing & $28(9.3)$ & $3(10.0)$ & \\
\hline \multicolumn{4}{|l|}{ Primary site (\%) } \\
\hline Right-side colon & $90(29.8)$ & $9(30.0)$ & 0.93 \\
\hline Left-side colon & $143(47.4)$ & $14(46.7)$ & \\
\hline Rectum & $65(21.5)$ & $7(23.3)$ & \\
\hline Other or missing & $4(1.3)$ & $0(0)$ & \\
\hline \multicolumn{4}{|l|}{ T-stage (depth of invasion) (\%) } \\
\hline $\mathrm{TI}-3$ & $185(6 \mid .3)$ & $14(46.7)$ & 0.28 \\
\hline $\mathrm{T} 4$ & $112(37.1)$ & $15(50.0)$ & \\
\hline Tx & $5(1.7)$ & I (3.3) & \\
\hline \multicolumn{4}{|l|}{ N-stage (lymphatic invasion) (\%) } \\
\hline No & $92(30.5)$ & $12(40.0)$ & 0.17 \\
\hline $\mathrm{N}+$ & $203(67.2)$ & $16(53.3)$ & \\
\hline $\mathrm{Nx}$ & $7(2.3)$ & $2(6.7)$ & \\
\hline \multicolumn{4}{|l|}{ MMR status (\%) } \\
\hline dMMR & $14(4.6)$ & $2(6.7)$ & 0.62 \\
\hline PMMR & $288(95.4)$ & $28(93.3)$ & \\
\hline \multicolumn{4}{|l|}{ Previous therapy (\%) } \\
\hline De novo metastatic disease & $240(79.5)$ & $20(66.7)$ & 0.11 \\
\hline Previous (neo) adjuvant therapy & $62(20.5)$ & $10(33.3)$ & \\
\hline
\end{tabular}


Table SI (Continued)

\begin{tabular}{|c|c|c|c|}
\hline & $\begin{array}{l}\text { Patients with evaluable } \\
\text { TILs }(N=302)\end{array}$ & $\begin{array}{l}\text { Patients with non- } \\
\text { evaluable TILs }(N=30)\end{array}$ & $P$-value \\
\hline \multicolumn{4}{|c|}{ Metastasectomy (\%) } \\
\hline+ & $58(19.2)$ & $9(30.0)$ & 0.16 \\
\hline- & $244(80.8)$ & $21(70.0)$ & \\
\hline \multicolumn{4}{|c|}{ No. of metastatic organs (\%) } \\
\hline 1 & $197(65.2)$ & $20(66.7)$ & 0.88 \\
\hline$\geq 2$ & $105(34.8)$ & $10(33.3)$ & \\
\hline \multicolumn{4}{|c|}{ Adjuvant chemotherapy (\%) } \\
\hline Negative & $76(25.2)$ & $6(20.0)$ & 0.53 \\
\hline Positive & $226(74.8)$ & $24(80.0)$ & \\
\hline \multicolumn{4}{|c|}{ Oligometastatic status (\%) } \\
\hline Yes & $135(44.7)$ & $12(40.0)$ & 0.62 \\
\hline No & $167(55.3)$ & $18(60.0)$ & \\
\hline
\end{tabular}

Note: Data are presented as total number (\%).

Abbreviations: dMMR, deficient mismatch repair; MMR, mismatch repair; pMMR, proficient mismatch repair; TILs, tumor-infiltrating lymphocytes.

Table S2 Adjusted hazard ratio of TIL for overall survival in different colorectal cancer subtypes excluding patients who received chemo/radiotherapy before surgery

\begin{tabular}{lllll}
\hline Subgroup & $\mathbf{N}$ & HR & 95\% Cl & P-value \\
\hline Metastasectomy & & & & $0.38-0.89$ \\
$\quad-$ & 194 & 0.58 & $0.28-2.65$ & 0.01 \\
+ & 46 & 0.86 & $0.58-1.92$ & 0.80 \\
Oligometastatic & 113 & 1.06 & $0.34-0.97$ & 0.85 \\
$\quad$ No oligometastatic & 127 & 0.58 & & 0.04 \\
No. of metastatic organs & & & $0.56-1.42$ & 0.63 \\
I & 153 & 0.89 & $0.20-0.78$ & 0.008 \\
$\geq 2$ & 87 & 0.39 & $0.41-0.90$ & 0.01 \\
Overall & 240 & 0.61 & \\
\hline
\end{tabular}

Abbreviations: HR, hazard ratio; TILs, tumor-infiltrating lymphocytes.

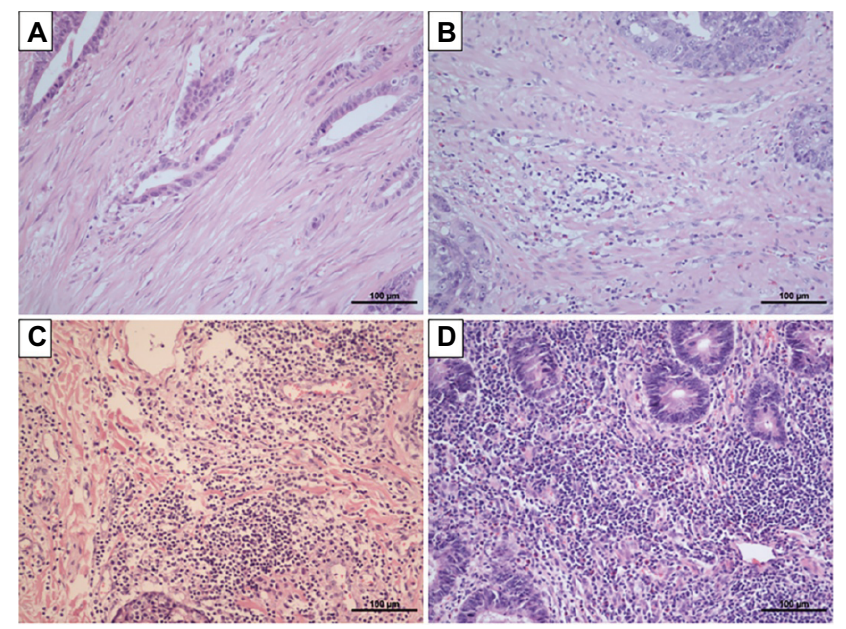

Figure SI Representative cancer specimens stained with H\&E demonstrating examples with absent (A), mild (B), moderate (C), or marked (D), of tumor-infiltrating lymphocytes, respectively. 
Cancer Management and Research

\section{Publish your work in this journal}

Cancer Management and Research is an international, peer-reviewed open access journal focusing on cancer research and the optimal use of preventative and integrated treatment interventions to achieve improved outcomes, enhanced survival and quality of life for the cancer patient.

The manuscript management system is completely online and includes

Submit your manuscript here: https://www.dovepress.com/cancer-management-and-research-journal

a very quick and fair peer-review system, which is all easy to use. Visit $\mathrm{http}: / / \mathrm{www}$.dovepress.com/testimonials.php to read real quotes from published authors. 